RUNNING TITLE: ADULT MENTAL HEALTH SERVICES AND BPD

\title{
Is contact with adult mental health services helpful for individuals with a diagnosable BPD? A study of service users views in the UK.
}

\begin{abstract}
Background: Previous research in the UK has suggested that individuals with a diagnosable borderline personality disorder (BPD) have often found contact with adult mental health services unhelpful. In 2003 UK government guidance outlined how services might address this issue. Since this guidance there has been a lack of research which seeks to understand services users’ experiences of services and provide information about how services might improve.
\end{abstract}

Aims: To explore the experiences of individuals with a diagnosis of BPD in accessing adult mental health services and to better understand which aspects of contact with services can be helpful or unhelpful.

Method: Nine service users with a diagnosable BPD were recruited through voluntary sector services in the north west of England. Semi-structured interviews were used and interview data was analysed using an inductive thematic analysis.

Results: Three themes were generated including 'The diagnostic process influences how service users feel about BPD’, 'Non-caring care', and 'Therapeutic relationships equate to good care'.

Conclusion: The participants' accounts identify a number of practical points which services could implement to improve the experiences of service users. Furthermore, these recommendations could be implemented with minimal cost to organisations.

Affiliations: The authors have no affiliation with funding organisations.

Keywords: borderline personality disorder, BPD, service users, thematic analysis. 
RUNNING TITLE: ADULT MENTAL HEALTH SERVICES AND BPD

Personality disorders are believed to affect around $10 \%$ of the general population (Alwin et al, 2006) and between 30\% and 50\% of psychiatric populations (Department of Health, 2009). Furthermore, in the United Kingdom (UK) borderline personality disorder (BPD) is reportedly the most commonly diagnosed personality disorder subtype within non-forensic mental health settings (NICE, 2009). The DSMIV diagnostic criteria for BPD includes dramatic fluctuations in mood, unstable interpersonal relationships, fear of abandonment, transient psychotic symptoms, problems with self-image and impulsive behaviours including self-harm and suicide attempts (APA, 1994).

However, significant concerns about the validity and reliability of personality disorder diagnoses have been described in the literature (Blackburn, 2006; Livesley, 2001; Zanarini et al, 2004). For example, the assessment and diagnosis of personality disorder is regarded as inaccurate and inconsistent across different services (Alwin et al, 2006). Furthermore, Pilgrim (2001) has suggested that within the UK National Health Service (NHS) personality disorder has been used as a “dustbin” diagnosis for clients who cannot be adequately described by other diagnostic categories.

Empirical research suggests that the personality disorder label can be stigmatising and that mental health professionals frequently hold negative views of clients identified as having a BPD and see them as less deserving of care than clients with an DSM axis I diagnosis (Cleary, Siegfreid \& Walter, 2002; Fraser \& Gallop, 1993; Lewis \& Appleby, 1988; Markham \& Trower, 2003).

In addition, due to a skills and knowledge deficit many mental health staff potentially lack an understanding about personality disorder and how to work effectively with clients meeting diagnostic criteria (NIHME, 2003). More specifically, Fallon (2003) reported that service users with a diagnosis of BPD experienced staff as unapproachable, overly restrictive or unable to adequately manage acute intrapersonal 
RUNNING TITLE: ADULT MENTAL HEALTH SERVICES AND BPD

distress. Previous research has reported that service users who have a diagnosis of BPD are often unaware of what it actually means (Castillo, 2000; Stalker, Ferguson and Barclay, 2005; Horn, Johnstone \& Brooke, 2007) and being diagnosed can be a demoralising experience (Horn, Johnstone \& Brooke, 2007). It appears that service users' concerns about the diagnosis may be justified as historically getting a diagnosis of personality disorder has represented a barrier to obtaining appropriate support from health services (Castillo, 2003; Stalker, Ferguson \& Barclay, 2005).

Individuals with a diagnosable BPD are reported to receive a substantial amount of input from services in the UK (Bender et al, 2001). However, adult mental health services are potentially unable to adequately meet the needs of this client group due to staff lacking the necessary skills and knowledge (NIMHE, 2004a). Given the prevalence of BPD the impact of poor services is likely to be far reaching. In response to this considerable deficit, national guidance for UK services, "Personality Disorder: No longer a diagnosis of exclusion’ (NIMHE, 2003), was published to address the issues.

A number of studies have previously been published on this topic, yet none of the studies published in recent years have focused specifically on the experiences of service users with a diagnosis of BPD in accessing adult mental health services. Furthermore, none of these studies are subsequent to the publication and implementation period for the national guidance (NIMHE, 2003) whereas the present study collected data between September and December 2009. The current study aimed to explore the experiences of individuals with a diagnosis of BPD in accessing adult mental health services and to better understand which aspects of contact with services which can be helpful or unhelpful. 
RUNNING TITLE: ADULT MENTAL HEALTH SERVICES AND BPD

\section{Method}

\section{Participants}

Nine participants, who had responded to a poster displayed in voluntary sector services in the North West of England, were recruited. The poster contained information regarding the aims of the study and the inclusion criteria and requested that volunteers contact the chief investigator if interested in taking part in a research interview. On initial contact candidates were provided with further details of the study and with their consent were asked a brief set of questions to ensure that they met the following inclusion criteria:

- Reported having a diagnosis of BPD

- A period of contact with general adult mental health services in the last 3 years

- $\quad$ Aged 18-65

- Resident in England

- Reported feeling mentally well enough to participate

The sample consisted of two males and seven females with ages ranging from 31 to 47 years. The total sample of nine participants is considered adequate for a qualitative study (Josselson \& Lieblich, 2003). All of the participants reported that they had other axis I and/or axis II diagnoses in addition to BPD. Eight of the participants described themselves as white British and one as British.

\section{Design}

A qualitative design was employed to explore service users' experiences. The findings of this study are positioned within the critical realist perspective (Robson, 2002) and are therefore seen as a co-construction by the participants and the researcher. 
RUNNING TITLE: ADULT MENTAL HEALTH SERVICES AND BPD

\section{Procedure}

The study was reviewed and approved by a local university ethics committee. Face to face semi-structured interviews were employed using an interview schedule which was not exhaustive and was flexibly implemented. Prior to commencing the interview members of an internet service-user group were consulted to ensure the interview schedule was an appropriate tool for understanding service users’ experiences. In addition, participants provided feedback about the interview content which was used alongside interview material to develop the interview schedule in an iterative process (Smith \& Osborn, 2008) and arising themes were explored as the study progressed. Interviews lasted 40-90 minutes, these were audio recorded and transcribed by the interviewer.

\section{Data analysis}

Transcripts were manually analysed using thematic analysis. An idiographic approach to data analysis was employed which involved an in-depth analysis of each interview transcript (Smith \& Eatough, 2007). Transcripts were read several times, annotated with initial ideas and coded in an inductive manner. The coding reflected the semantic and latent content of units of data and ensured that the identified themes reflected participants’ accounts overall (Braun \& Clarke, 2006). Initial codes were collated and arranged into clusters to reflect potential themes which were then sorted into fewer, broader and more interpretive themes. This resulted in the generation of three final themes. The themes were developed on the basis of their prominence in the data including numerous references to the theme across a variety of transcripts. Theme development was descriptive and interpretive and therefore the analytic process represents an interaction between the researcher and the data.

Several procedures, described by Yardley (2008), were implemented to increase the trustworthiness of the findings. These included a reflective diary 
RUNNING TITLE: ADULT MENTAL HEALTH SERVICES AND BPD

documenting how the researcher's own experiences, values and feelings could impact on the analytic process. In addition, a record was kept documenting theme development and how decisions were made about the analytic process. Two of the transcripts were read by one of the co-authors who independently generated and corroborated the developing themes. Finally, negative case analysis, a process involving the analysis and reporting of experiences that differed from the experiences of the majority, was incorporated.

\section{Results}

Three interconnected themes were developed from the participants’ interviews. These capture the experiences of the participants in accessing adult mental health services. The three themes are described below and are accompanied by participant quotes to illustrate them. The theme titles were developed by the researchers. Pseudonyms have been used and quotes have occasionally been minimally amended to assist with ease of reading.

\section{The diagnostic process influences how service users feel about BPD}

The participants indicated that the way they were told about their diagnosis influenced how they felt subsequently about BPD. BPD was viewed by many of the participants as an arbitrary label which they had attracted after many years of accessing services and after having already exhausted a host of axis I diagnoses. Furthermore, diagnosis was described as arising as a result of participants experiencing some change in their care which meant that they came into contact with a new staff member or a new service.

It was when I was changing psychiatrist...because I'd moved areas...I had to go into hospital and the psychiatrist said “oh you're not psychotic, you're not this, you're not that”, and he sort of plucked it from the air basically, “oh borderline personality disorder” (Rose). 
RUNNING TITLE: ADULT MENTAL HEALTH SERVICES AND BPD

There was considerable variability in how participants had discovered they had a diagnosis of BPD. Some participants reported being told in an insensitive manner which allowed little opportunity for discussion about what the diagnosis meant. These participants seemed to feel less positive about BPD than those who felt that the diagnostic process had been helpful and handled well by staff. One participant reported a diagnostic process which was strikingly collaborative and allowed space to discuss BPD and what it meant.

He drew my attention to it [borderline personality disorder], suggested I maybe went away and get some ideas about what it is and what I thought about it (Johnny).

The participants appeared to place great value on being given information about BPD and there was a strong sense that services in many cases were failing to provide this. As a consequence participants reported having a limited understanding of what the diagnosis meant.

I still don't understand what it is, I still don't, I know I'm not a personality disorder, I'm borderline personality disorder which is different (Eric).

In light of a paucity of information provided by health professionals, participants reported relying on the internet for this. Depending on the nature of information that services users came across on the internet this had the potential to impact on how they perceived the diagnosis and their level of optimism about recovery. It appeared that this was particularly the case in the absence of a trusted mental health professional to discuss the information with.

Optimism about how effective treatment for BPD might be and the likelihood of recovery were further factors seeming to impact on the way in which participants 
RUNNING TITLE: ADULT MENTAL HEALTH SERVICES AND BPD

perceived their diagnosis. For example, Victoria had been diagnosed with BPD by her psychiatrist, and she reported him stating “there’s no cure”, which understandably led her to feel distressed. Conversely, Elizabeth had been given the diagnosis by a psychologist within the context of therapy and had then gone on to complete therapy successfully. In addition, she reported staff being optimistic about her treatability and so felt that the diagnosis had played an important part in her treatment as it gave her “something to recover from”.

However, a number of the participants felt that staff were "cagey” about disclosing their diagnosis or they reported that the diagnosis had been recorded in their files for years but they had not been told until much later. All participants expressed a strong desire for services to be more honest about BPD.

\section{Non-caring care}

Although the participants were accessing NHS mental health services, all described experiencing significant gaps in care and felt that services were disjointed and unreliable.

I just get a letter every year, "we have still got you on file, we will get someone to come and visit you some time”, but they don’t (Emma). In addition, poor communication meant that participants could feel unsupported, in "limbo", and often did not know what to expect from services. Participants believed that the lack of service presence was due to the reluctance or inability of staff to work with them because they were perceived as challenging, complex or high risk.

When somebody presents a huge spectrum of problems they don't know how to deal with them so in the end they just wash their hands of them and leave them to their own devices until a crisis happens (Mercy).

Furthermore, services were described as being reactive to issues of risk and to demonstrate a 'knee-jerk' response if participants were viewed as being at risk of self- 
RUNNING TITLE: ADULT MENTAL HEALTH SERVICES AND BPD

harm. For example, Bob reported attending Accident and Emergency departments several times and believed that he was only admitted to inpatient care when he used the phrase, “I’ve got an immediate intention now to harm myself”. Services were perceived as defensively admitting participants to inpatient care to prevent self harm. However, the participants' account suggested that once risk had subsided services appeared to be less concerned about the issues underlying the risk such as participants' “distress” and “despair”.

Participants reported that services increased input when there was an obvious risk of self-harm or suicide. However, one participant, who had sought out help as he was experiencing intrusive and distressing thoughts about harming others, described how services withdrew while they decided which service would be the most appropriate to intervene. A further participant described how a therapist he had been working with discharged him because he had been in crisis and presented as too risky for staff to continue to engage with. The withdrawal of services at this crucial time was viewed as a "total contradiction” and represented a removal of support when it was needed most. Overall, participants felt that services lacked the skills to work therapeutically with risk and focused on risk reduction rather than risk prevention.

Moreover, participant's described feeling defined by the BPD label and believed that once they got the diagnosis staff interpreted their difficulties solely in terms of BPD. They reported no longer being seen as unwell or distressed but as "difficult”.

As soon as I do something wrong or react to something or, you know, have a blip or whatever, it’s “oh it’s her personality disorder” (Rose).

Symptoms such as psychotic experiences were no longer viewed as genuine and were dismissed by staff as not "real”. 
RUNNING TITLE: ADULT MENTAL HEALTH SERVICES AND BPD

Overall, the participants' accounts suggested that services could be inaccessible and unhelpful. The majority of participants believed that specialist services which employed staff with skills and knowledge specific to BPD would be ideal and would counteract feelings of hopelessness associated with the diagnosis. Many of the participants expressed a desire for more intensive interventions such as psychological therapies as well as low key, easily accessible services such as day centres and groups.

Although many of the participants reported unhelpful experiences of services there was an indication that pockets of more positive clinical practice were taking place. For example Johnny reported that one staff member, who had identified that Johnny had a diagnosable BPD, ensured that he saw the same worker consistently even though this was contrary to the way the service usually operated. However, it seemed that experiences of good practice tended to result from chance meetings with a knowledgeable staff member rather than being an inherent part of the service culture. Participants were acutely aware that there was a paucity of services specifically for BPD and none were aware of the specialist services for personality disorder such as those recommended in the government guidance for services (NIMHE, 2003).

\section{Therapeutic relationships equate to good care}

Participants had varying degrees of contact with mental health professionals from a wide range of disciplines. The participant's accounts clearly communicated that therapeutic relationships with the staff were of great importance and that a helpful therapeutic alliance reflected good care. Many participants described particular members of staff who had taken the time to listen or had treated them like "a person rather than...a case number” (Rose). Positive experiences ranged from contact with staff who had actively listened to the participants through to intensive psychological 
RUNNING TITLE: ADULT MENTAL HEALTH SERVICES AND BPD

therapy. Encounters with staff who wanted to engage and were willing to try and fit care around service users in a flexible manner were greatly appreciated and had the potential to increase self-esteem and hope for participants. For example, Ellis described her key worker as “one of the few people in my life that I actually trust”.

All of the participants reported some positive encounters with staff. However, their accounts also suggest that at times contact with staff was fraught with difficulties. The participants described some members of staff who made them feel “criticised” and "blamed”, lacked compassion, and had little interest in listening to them. When participants needed to talk, some staff were reported to respond unhelpfully, for example by saying “I don’t want to know” (Rose). This type of response was reported to amplify feelings of “emptiness” and alienation for participants who were often already distressed.

Moreover, staff were described as being focused on practical help, such as coordinating care. Although this was seen as valuable and necessary, the participants expressed the need for more psychological and emotional support in terms of formal therapy or "just someone to talk to" (Emma). There was a sense that what services provided was superficial and a poor match with the levels of distress the participants experienced.

Participants were aware that services were under pressure and under-resourced and they recognised that this influenced the quality of care they received. The need to build trust as the basis for an effective therapeutic alliance takes time and the participants felt that staff were often either unwilling to dedicate time in this way or were unable to so due to a lack of resources. However, participants wanted staff to place an emphasis on building an effective therapeutic alliance with them. Johnny was asked what he wanted from the therapeutic relationship, his response is below. 
RUNNING TITLE: ADULT MENTAL HEALTH SERVICES AND BPD

To take time to get to know me, understand me, feel that they accept me...to take that time to appreciate the situation and give me time to trust them.

Furthermore, participants described a real struggle in admitting that they needed help and in opening up and being honest with staff about their distress. Once they decided to seek help and put their trust in staff they felt that there was a risk that their fears of "abandonment” and "rejection” would be confirmed if staff then withdrew care. Services were likened to a "blunt” tool which could not effectively respond to service users' needs and withdrew care when things got difficult.

I was a bit rude really...so he’s said he’s not going to deal with me, he’s not going to work with me (Eric).

There was a strong sense within the participants' accounts that staff lacked knowledge and understanding about BPD. Indeed, some of the participants believed that the lack of skills and knowledge explained why staff were unable to maintain helpful therapeutic relationships with them, particularly when there were issues of risk.

\section{Discussion}

This study aimed to explore the experiences of service users identified as having BPD in accessing adult mental health services. The sample size was relatively small yet sufficient for a qualitative study of this type. The purpose of the study was not to create findings which could be widely generalised, but to generate rich data which provide examples of people's experiences of services and highlights how practice may be improved when working with this client group.

Previous research with service users (Rogers \& Dunne, 2011) and with mental health staff, highlights negative staff attitudes towards service users with a diagnosable personality disorder (Cleary, Siegfreid \& Walter, 2002; Fraser \& Gallop, 1993; Lewis \& Appleby, 1988; Markham \& Trower, 2003). Prejudice towards those 
RUNNING TITLE: ADULT MENTAL HEALTH SERVICES AND BPD

with a diagnosable personality disorder is likely to prevent staff from engaging service users in helpful therapeutic relationships. In the current study the participants felt that some of the basic elements of the therapeutic alliance were often lacking in their interactions with staff. Indeed, participants suggested a need for an increased emphasis on the therapeutic relationship and skills such as listening and communication. Furthermore, participants highlighted the importance of staff who were consistent and who would stay engaged during difficult periods, such as during times of crisis when risk might be higher and when participants most needed the staff who knew them best. In addition, the participants felt that contact with services could be improved if there was a greater focus on the distress which lay beneath issues of risk, rather than the emphasis being solely on reducing the risk itself.

To this end services and staff should consider how to invest more time in building therapeutic relationships and in providing a safe space for clients to discuss their difficulties whether this be within formal therapy or in the form of low-key support. Clinical skills such as active listening form part of every mental health professionals' training, so it is likely that a broad range of staff would be able to undertake this work. Changing care to emphasise the therapeutic relationship is likely to be inexpensive yet does not preclude risk assessment and management. Moreover, it has the potential to reduce the number of people requiring access to expensive services such as inpatient care or specialist services for personality disorder.

Furthermore, the findings on diagnosis described in this study resonate with those reported in previous research. For example, a number of previous studies have described how being diagnosed with a personality disorder can be a distressing experience for participants (Castillo, 2003; Fallon, 2003; Horn, Johnstone and Brooke, 2007; NIMHE, 2003; Stalker, Ferguson and Barclay, 2005). However, in the current study the participants' accounts emphasised that the way that the diagnosis 
RUNNING TITLE: ADULT MENTAL HEALTH SERVICES AND BPD

was communicated significantly impacted on how they adjusted to and related to it subsequently. Therefore, the value of a collaborative and sensitive process of diagnosis which enables the person to feel optimistic about recovery may be invaluable to some service users.

In addition, the impact of professionals being open with service users about their diagnosis and providing accurate information should not be under-estimated. The current study found that the service users were often relying on the internet to find out about their diagnosis and that the information they found there could influence their perceptions of what the diagnosis meant. This finding further highlights the importance of services providing appropriate information in a constructive way so as to prevent service users developing an inaccurate perspective based on unreliable sources. Moreover, the findings suggest that despite the negative connotations, some service users may prefer to know what their diagnosis is and if given along with accurate information this has potential to form an important part of the recovery process.

Although based on a small sample the findings of this study suggest that some service users with a diagnosable BPD’s experience of accessing adult mental health services in the UK has remained unchanged since the implementation of the government guidance (NIHME, 2003). Indeed, for some service users a diagnosis of BPD may continue to represent a barrier to accessing adequate care from mainstream mental health services. Services in the UK are now required to be inclusive of clients with a diagnosable personality disorder (DH, 2007) yet the pervasive attitudes towards the diagnosis may result in implicit exclusion or contact with services which is not therapeutic or even iatrogenic.

There is no denying that major service reform and large scale structured training packages, which would lead to an attitude shift and greater knowledge and 
RUNNING TITLE: ADULT MENTAL HEALTH SERVICES AND BPD

skills amongst staff, could significantly improve service users' experiences. However, the importance of smaller, more subtle changes made by services locally and by individual staff members, such as focusing on how the diagnosis is reached and communicated, or taking the time to listen to service users in distress, should not be overlooked.

\section{Conclusion}

This study has identified a number of inexpensive points for service development which may enable services to better meet the needs of those with a diagnosis of BPD. The accounts of the participants in the current study highlighted the importance of sensitive and collaborative diagnosis. Furthermore, the willingness of staff to engage them in meaningful and consistent therapeutic relationships was seen to be of great value. Finally, the impact of the quality of interactions with service users, such as taking the time to listen and understand clients' distress, may have a far reaching impact.

\section{References}

APA (American Psychiatric Association). (1994). Diagnostic and statistical manual of mental disorders. 4th ed. Washington, D.C.: American Psychiatric Association.

Alwin, N., Blackburn, R., Davidson, K., Hilton, M., Logan, C., \& Shine, J. (2006). Understanding Personality Disorder. The British Psychological Society: Leicester.

Bender, D.S., Dolan, R.T., Skodol, A.E., Sainslow, C.A., Dyck, I.R., McGlashan, T.H., Shea, M.T., Zanarini, M.C., Oldham, J.M., \& Gunderson, J.G. (2001). Treatment utilisation by patients with personality disorders. American Journal of Psychiatry, 158, 295-302.

Blackburn, R. (2006). What is personality disorder? In M.J. Sampson, R.M. 
RUNNING TITLE: ADULT MENTAL HEALTH SERVICES AND BPD

McCubbin \& P. Tyrer, (eds). Personality disorder and community mental health teams: A practitioner's guide (pp. 21-40). West Sussex, England: John Wiley \& Sons.

Braun, V., \& Clarke, V. (2006). Using thematic analysis in psychology. Qualitative Research in Psychology, 3, 77-101.

Castillo, H. (2000). Temperament or trauma? Users’ views on the nature and treatment of personality disorder. Mental Health Care, 41(2), 53-58).

Castillo, H. (2003). A dangerous diagnosis. Mental Health Today, 27-30.

Cleary, M., Siegfried, N., \& Walter, G. (2002). Experience, knowledge and attitudes of mental health staff regarding clients with a borderline personality disorder. International Journal of Mental Health Nursing, 11(3), 186-191.

Department of Health (2007). Mental Health Act. Retrieved from http://www.legislation.gov.uk

Department of Health. (2009). Recognising complexity: Commissioning guidance for personality disorder services. Retrieved from http://www.dh.gov.uk/

Fallon, P. (2003). Travelling through the system: The lived experience of people with borderline personality disorder in contact with psychiatric services. Journal of Psychiatric and Mental Health Nursing, 10(4), 393-400.

Fraser, K., \& Gallop, R. (1993). Nurses' confirming/disconfirming responses to patients diagnosed with borderline personality disorder. Archives of Psychiatric Nursing, 7(6), 336-341.

Horn, N., Johnstone, L., \& Brooke, S. (2007). Some service user perspectives on the diagnosis of borderline personality disorder. Journal of Mental Health, 16(2), 255-269.

Josselson, R., \& Lieblich, A. (2003) A framework for narrative research proposals in Psychology. In Josselson, Ruthellen, Lieblich, Amia, McAdams, \& Dan P. 
RUNNING TITLE: ADULT MENTAL HEALTH SERVICES AND BPD

(Eds.) (2003). Up close and personal: The teaching and learning of narrative research. The narrative study of lives. (pp. 259-274). Washington, DC, US: American Psychological Association.

Lewis, G., \& Appleby, L. (1988). Personality disorder: The patients psychiatrists dislike. British Journal of Psychiatry, 153, 44-49.

Livesley, W.J. (2001). Conceptual and taxonomic issues. In W.J. Livesley, ed. Handbook of personality disorders: Theory, research, and treatment (pp.339). New York: Guilford Press.

Markham, D. (2003). Attitudes towards patients with a diagnosis of 'borderline personality disorder': Social rejection and dangerousness. Journal of Mental Health, 12(6), 595-612.

NICE (National Institute for Health and Clinical Excellence) (2009). Borderline personality disorder: treatment and management. Retrieved from http://www.nice.org.uk/Guidance/CG78/NiceGuidance/pdf/English

NIHME (National Institute for Mental Health in England) (2003). Personality disorder: No longer a diagnosis of exclusion. London: Department of Health.

Pilgrim, D. (2001). Disordered personalities and disordered concepts. Journal of Mental Health, 10(3), 253-265.

Robson, C. (2002). Real World Research. $2^{\text {nd }}$ ed. Oxford: Blackwell Publishers.

Rogers, B, \& Dunne, E. (2011). They told me I had this personality disorder ... All of a sudden I was wasting their time’: Personality disorder and the inpatient experience. Journal of Mental Health, 20, 226-233.

Smith, J.A. \& Eatough, V. (2007). Interpretive Phenomenological Analysis. In E. Lyons, \& A. Coyle, eds. Analysing qualitative data in psychology (pp.35-50). London: SAGE. 
RUNNING TITLE: ADULT MENTAL HEALTH SERVICES AND BPD

Smith, J.A., \& Osborn, M. (2008). Interpretative phenomenological analysis. In

J. Smith, ed. Qualitative psychology: A practical guide to research methods. $2^{\text {nd }}$ ed. (pp. 53-80). London: SAGE.

Stalker, K., Ferguson, I., \& Barclay, A. (2005). 'It’s a horrible term for someone’: service user and provider perspectives on 'personality disorder'. Disability and Society, 20(4), 359-373.

Zanarini, M.C., Frankenburg, F.R., Vujanovic, A.A., Hennen, J., Reich, D.B., \& Silk, K.R. (2004). Axis II comorbidity of borderline personality disorder: description of 6-year course and prediction to time-to-remission. Acta Psychiatrica Scandinavica, 110(6), 416-420.

Yardley, L. (2008). Demonstrating validity in qualitative psychology. In J. Smith, ed. Qualitative Psychology: a practical guide to research methods. $2^{\text {nd }}$ ed. London: SAGE. 